\title{
Analysis of Urban Planning Changes by Biological Metaphor (Case of DG-ABC Model)
}

\section{Mohammad Rahim Rahnama1, Alireza Bidkhori*2, Amirali Kharazmi ${ }^{3}$}

1Associate Professor of Geography Department, Ferdowsi University of Mashhad(FUM)

*2 PhD Candidate of Urban Geography, Ferdowsi University of Mashhad(FUM), International Campus, Faculty Member of Kheradgarayan Motahar Higher Education Institute 3PhD Candidate of Urban Geography, Ferdowsi University of Mashhad(FUM), International Campus

Corresponding Author E-mail: alirezabidkhori@yahoo.com

Received: 24 October 2018, Revised: 05 December 2018, Accepted: 20 December 2018

\section{ABSTRACT}

Urban concept has been defined as an autonomous and complex system. Despite the overwhelming differences between urban growths around the world, their growth still follows some universal mechanisms. Darwin's theory of evolution might have enlightened an innovative view to perform the research of urban development; as a consequence, many researchers have been trying to link their research to the biological metaphor of urban evolution. In the context of an analogy to biology, urban concept can be seen as 'organic' and many concepts from biology can be borrowed to explain the 'uncertainty' and 'relativity' of urban growth processes. Urban DNA is one of the innovative concepts, which has been used to describe the unique characteristics of urban concept and the common fundamental elements of each urban area. The main ideas of applying the concept of urban DNA involve identifying the key factors/ metrics which reflect urban characteristics, hence allowing the understanding of the cities' characteristics with urban DNA. This would allow, among other things, to identify or propose optimal urban form and 'smooth' transition of growth patterns and the characteristics of urban from a suboptimal urban form. As Silva (2004) explained, the possibility of defining this 'key' (DNA) for each region seems to be of great significance in the planning studies. It allows for the understanding of how the different elements that constraint the functioning of urban system progress and constrain different regions, and what function they should have in shaping future scenarios. This research explores the theory aspects of urban DNA, and makes an attempt to link this concept with an integrated urban growth model (DG-ABC). The simulation results of a pilot study are analyzed in the context of the biology analogy in order to test the possibility of deriving urban DNA from DG-ABC model, by doing so we hope to understand how the key factors and parameters influence the formation of urban patterns, and hence therefore allowing developing optimal solutions to urban growth problems.

Keywords: Urban Planning Changes, Biological Metaphor, DG-ABC Model, Urban Patterns. 


\section{Introduction}

The DNA of Our Region: The Biological Metaphor of Urban Evolution

In the past decades, many researchers (Langton, 1986; Openshaw and Openshaw , 1997; Batty and Longley, 1994; Batty and Xie, 1996; Webster, 1996; Silva, 2001; Silva and Clarke, 2002; Silva, 2004; Silva and Clarke, 2005; Silva, 2006; Nicholas and Clarke, 2006; Caglioni et al. 2006; Li et al., 2008; Wilson, 2008) have explored urban DNA as a way of linking urban models with the biological metaphor of urban evolution.

Urban growth in evolving systems should resemble two-layer aggregates of spatial and a-spatial factors; this two-layer dynamics (spatial and a-spatial) indicates the characteristics of different urban growth processes. Therefore, urban DNA should consist of a-spatial and spatial structures to reflect the a-spatial and spatial dynamics of urban growth. In order to rationalize the nature of urban morphologies and analyze how the spatial and a-spatial metrics lead urban development to different ways, this part of research makes an attempt to clarify the structure and organization of urban DNA.

\section{The A-Spatial Organization of Urban DNA}

Generally, there are two kinds of key factors influencing urban growth in socioeconomic context (as shown in Figure 1). One kind is related to the driving forces of urban growth, which are the endogenous factors with slow rate of changing because most of them originate from the whole historical processes of urban development, such as the historical data of urban growth; the cultural/social or religion factors which have direct influences to urban entities' behaviors, and governments' policy making. The other group of factors are related to the control elements of urban growth, which can be adjusted by urban planners to take urban develop to a desired direction.

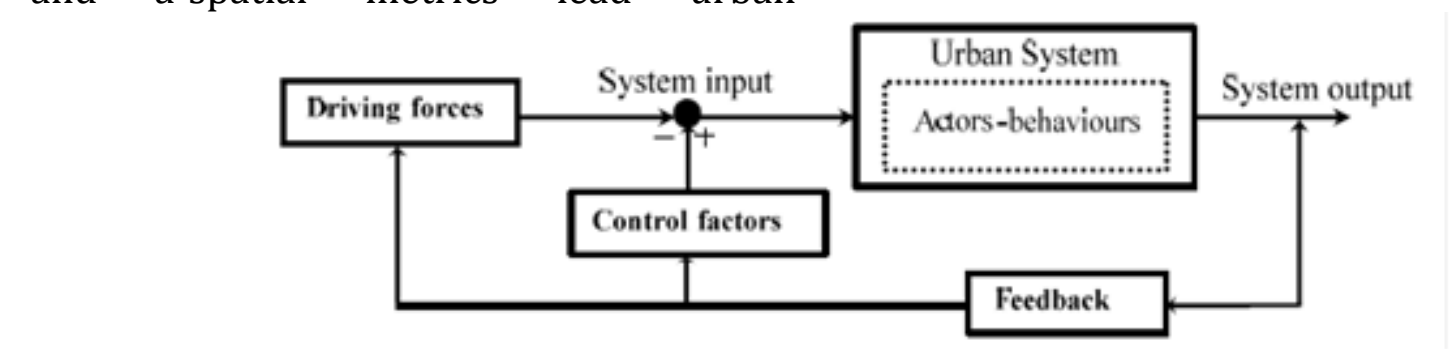

Figure 1. The control theory view of urban system

It is reasonable to think the a-spatial part of urban DNA structure should be derived from both the two kinds of key factors, which have the ability to reflect and represent the key driving forces of urban growth. At the same time the change of the a-spatial structure of urban DNA may result in fundamental transformation of urban growth processes, because they represent some a-spatial characteristics of urban growth. To some extent, we think urban system work like a control system. According to control theory (Franklin et al., 2002) urban planners can manipulate the control factors of urban system to obtain the desired effect on the output of the system.

Therefore, an urban DNA should include both driving factors and control factors. Understanding the a-spatial organization of urban DNA will help urban planner and researchers effectively control the key 
issues in order to pull the social and spatial agendas in the same direction. This has led to recent attempts to analyze urban growth using biological evolutionary views.

\section{The Spatial Organization of Urban DNA}

Apart from the a-spatial organization of urban DNA, the model also includes spatial organizations of patterns, and particularly of geo-spatial factors. Urban growth's spatial attributes are quantifiable for each city. A set of spatial metrics can be developed to describe each attribute and represent the dynamics of the city, but spatial metrics should be selected and analyzed according to the context of each application. In many urban models, some spatial attributes have emerged as the key characterizations of urban growth and sprawl.

Classification and calibration are the two good approaches to identify the metrics of urban spatial structure that are commonly associated with growth. We can take the link of urban DNA with SLEUTH model as an example. With the calibration processes we can get a set of spatial coefficients (Dispersion, breed, spread, slope, road gravity) which govern four types of urban growth in this system. As Silva and Clarke (2004) suggested, we can take the five coefficients as the urban DNA of our regions.

Classification of data is another way to distinguish the spatial metrics and its manifestations in the urban process. For example, the maximum likelihood classification and the scaling method in remote sensing have been used to characterize urban growth, so researchers tend to point that these are the best classifications to represent certain patterns/processes.

\section{The Conceptual Definition of Urban DNA}

As with biological evolution, urban evolution can be seen as a long-term aggregate effect which involves a combination of various evolutionary components such as road growth, buildings and land use. Barthe'lemy and Flammini, (2008) stated that the selforganized pattern of streets emerges as a consequence of the interplay of the geometrical disorder and the local rules of optimality. Beyond the economic, demographic and other exogenous forces that shape a city, we can think urban as an organic and self-organized system. As Barthélemy described: "cities are not just the result of rational planning - in the same way that living organisms are not simply what is in their genetic code". As shown, as an example in a sketch design, in Figure 2, A-F represent different cities and the lines between them can be seen as the road networks. From the view of urban morphology, urban concept evolves like an organism or embryo, even in the past the "unplanned" city evolved based on some simple universal mechanisms despite significant cultural and historical differences.

The evolution nature of urban growth, especially the urban DNA is a view borrowed from the mechanisms in biological research such as the natural selection, crossover, mutation and hybrid of genes. These concepts can be applied to urban studies, for instance in what regards the application of urban plans for different cities. Unlike biological variation and natural selection, in the urban context, in many cases, there will be some deliberate purpose applied to individual instances of variation and selection (Marshall, 2007). 


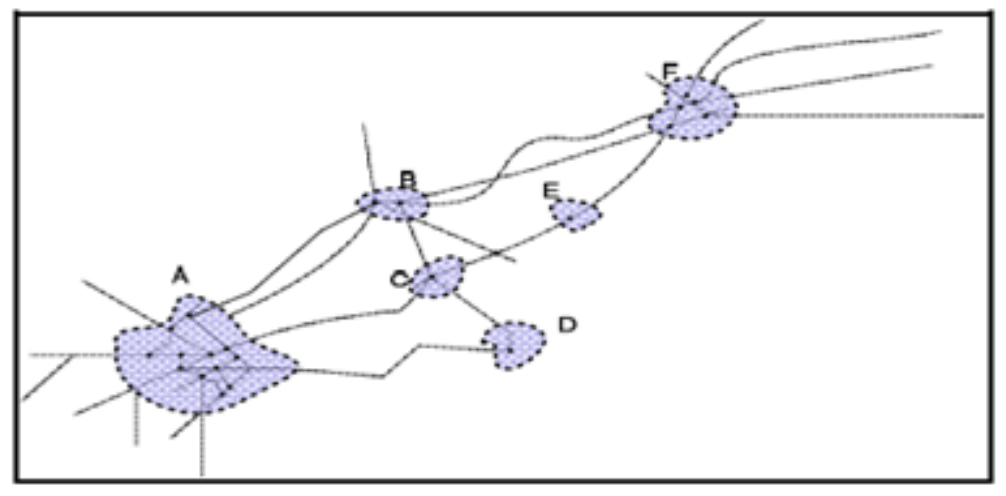

Figure 2. The organism/organic view of urban system

Admittedly, it is difficult to define the generic DNA set for all urban analysis, not only because urban is a complex systemthere are so many variables which could influence urban growth process- but also different DNA might be derived for different applications or specific perspective view. In spite of that, it is possible for us to define a general structure of urban DNA, as shown in Figure 3.
Generally, the urban DNA integrates both spatial and a-spatial 'genes' of city. In Figure 3, ' $A$ ' stands for the a-spatial organizations of urban DNA, 'S' stands for the spatial organizations of urban DNA, and the 'interactions' includes the interactions between spatial and a-spatial organizations. These 'gene' may be structured in different ways to represent different DNA for different cities.
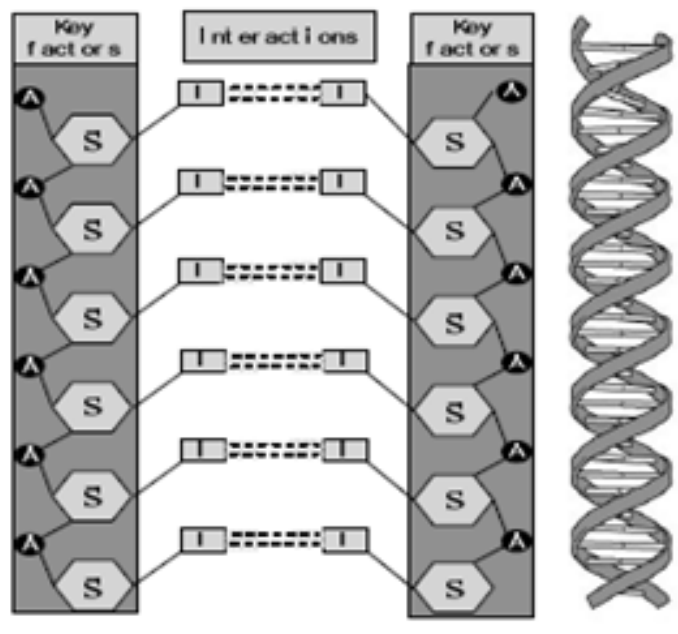

Figure 3. General structure of urban DNA

\section{Urban DNA with Dg-Abc Model}

DG-ABC model is an integrated dynamic urban model based on the loose-coupling of an agent-based model and a CA model (SLEUTH) for the simulation of urban growth phenomena. There are three kinds of agents in the model: resident, government and property developers. The behaviors of agents are regulated by Theory of Planned Behavior (Ajzen, 1985) and optimized by GA.

In DG-ABC model, residential growth is taken as the main driver for urban growth. From the simulation result, we can find the influences of social-economic factors to 
residential areas according to resident's social groups (mainly income levels) and land price. Among the factors, land price can be seen as the overlaid attribute of both spatial (geographical location) and aspatial factors (economic development level and residence income level of the studied city). According to Figure 1, it is obviously that one should consider land price as one of the driving forces of urban sprawl from the view of control theory, since it is one of the important reasons for residential sprawl to suburbs as many residents may not able to afford high price housing. Therefore, we think land price is an important factor to construct the aspatial structure of Urban DNA based on DG-ABC model.

The behaviors of agents in DG-ABC are regulated by a genetic algorithm and the Theory of Planned Behavior. According to $\mathrm{TpB}$, the behaviors of resident agents are determined by their intention $(I)$ and actual behavioral control (AbC).

$A b C^{a}=\delta_{1} \cdot a \cdot E_{\text {traffic }}+\delta_{2} \cdot b \cdot E_{\text {environment }}+\delta_{3} \cdot c \cdot E_{\text {convenience }}+\varepsilon_{t i j}$

Therefore, one of important steps in modeling processes of $\mathrm{DG}-\mathrm{ABC}$ is to derive the three weights (i.e. Etraffic, Environment and Econvenience) by GA in order to calculate three important utilities (traffic utility environment utility and convenience utility) in $\mathrm{TpB}$ formulation as a way to decide resident's decisions on location choice. The three weights are regarded as actual behavioral control factors when DG-ABC calculates TpB utility. Because they differentiate residents' choices on different locations depending on the locations' spatial attributes instead of residents' socialeconomic status (i.e., their income level, family structure). Again, when link DGABC's a-spatial factors back to Figure 1, the three weights (or the three utilities) can be seen as the control factors from control theory view. As stated in part 3.1, the aspatial structure of urban DNA should include both driving factors and control factors. Therefore, in this research we take land price, and the three utilities (traffic utility environment utility and convenience utility) as the a-spatial structure of urban DNA.

In this research DG-ABC is applied to a pilot study that is mainly based on a hypothetical city called Demo City (Clarke, 2008). The spatial dynamics of urban growth is simulated by SLEUTH, therefore we think the five spatial attributes (Slope, land use, excluded, urban, transportation, hill shade) composing the spatial organization of urban DNA in DG-ABC model. As a result, apart from the a-spatial attributes, the urban DNA has included some spatial organizations (the five spatial attributes) and a-spatial organizations such as the land price, and traffic utility (Tutility), convenience utility (C-utility) and environment utility (E-utility).

The urban DNA is illustrated in Figure 4 with three sample 'genes' in DG-ABC model. Urban DNA's related spatial attributes are the five spatial attributes, and its related a-spatial attributes include the three utilities and land price which reflect the socio-economic properties of the cells. In this Figure, 'A' represents the A-spatial structure of urban DNA, surrounded by the spatial organizations of urban DNA, the five spatial attributes (S: Slope, L: land use, E: excluded, U: urban, T: transportation, $\mathrm{H}$ : hill shade). Table 1 presents the values of the three 'genes'. For example, in the first 'gene' the spatial structure values are SLEUTH: 1, 4, 0, 255, 1 , and 12 , the utilities and land price are $89.2,91.12,80.80,85$, respectively. 


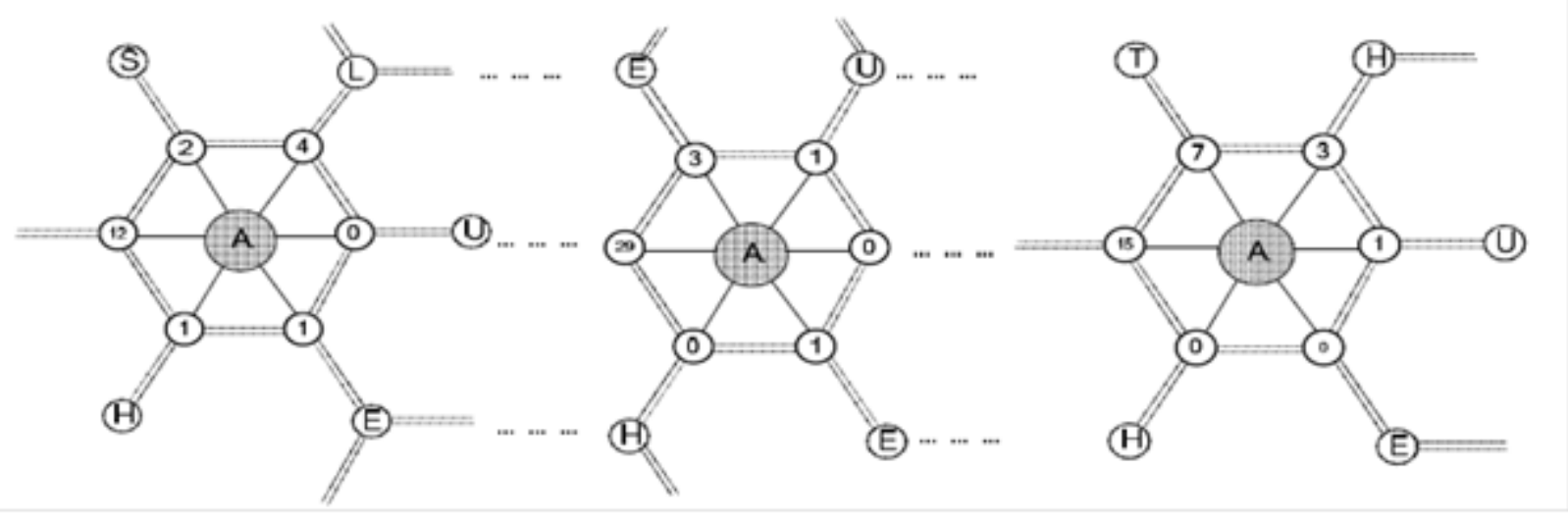

Figure 4. Urban Genes based on DG-ABC model

Table 1. Sample of the spatial and a-spatial attributes related to cells

\begin{tabular}{|l|l|l|l|l|l|l|l|l|l||}
\hline \multicolumn{9}{|c|}{ Cell related Spatial attributes } & \multicolumn{3}{l||}{ Cell related A-spatial attributes } \\
\hline S & L & E & U & T & H & T-utility & C-utility & E-utility & land price \\
\hline 2 & 4 & 0 & 255 & 1 & 12 & 89.42 & 91.12 & 80.80 & 85 \\
\hline 3 & 1 & 0 & 255 & 0 & 17 & 97.22 & 33.90 & 56.39 & 45 \\
\hline 7 & 3 & 1 & 0 & 0 & 15 & 45.24 & 56.32 & 10.01 & 27 \\
\hline
\end{tabular}

It is thought that such urban DNA would allow an understanding of the differences and similarities among different urban areas of similar or different ranges and hierarchies. The changes either in the values of spatial structure or a-spatial structure of urban DNA will result in fundamental changes of urban growth. For example, Figure 5 demonstrates the comparison of urban growth when changes happened to the values of aspatial structure (Etraffic, Environment and Econvenience). Figure 5 (a), (b) and (c)
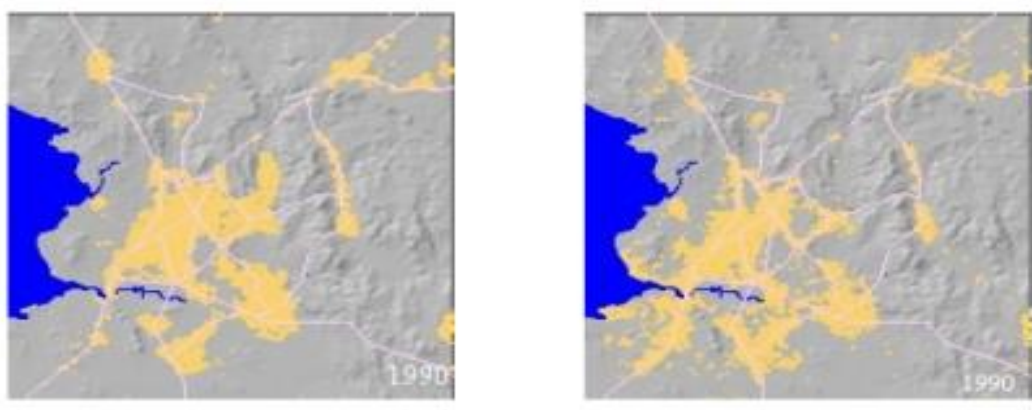

depicts the outcomes of three different growths respectively in DG-ABC model when the weights' values have large changes: city center sprawl, environment driven growth and growth along traffic networks. For example, there are more urbanized cells in the city center in Figure 5(a) and Figure 5(b) demonstrate more urbanized cells plus the water areas. Figure 5 (c) shows there are more urbanized cells along road network, particularly in the junction areas of road networks.

Figure 5. Comparisons of different spatial weights

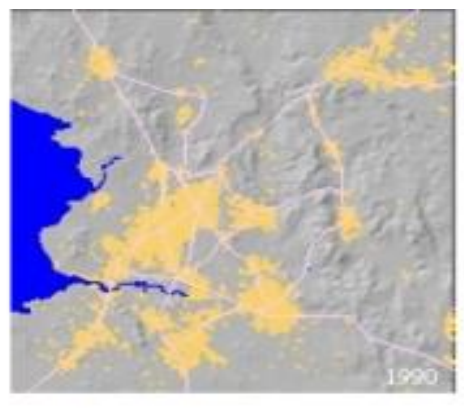


Table 2, The calibration results of the weights (Etraffic, Environment and Econvenience) in the DGABC model in the pilot study.

Table 2. The weights of resident agents

\begin{tabular}{llll}
\hline Coefficients & Convenience & Traffic & Environment \\
\hline & 0.564 & 0.287 & 0.149 \\
\hline
\end{tabular}

The three weights are global weights to the DG-ABC model; they are derived from the historical urban growth and manifest how the three aspects exert global influences on urban growth to all the residents. This is also a reason why we take it as a part of urban DNA, as to a specific city, although residents vary according to their social-economic status but their weights are the same. In other words, every city has a unique urban DNA which demonstrates the characteristics of the city on both spatial and a-spatial aspects.

\section{CONCLUSIONS}

From the analysis above, it can be concluded that Urban DNA is a good analytical approach that can contribute to defining future analysis of urban development and identifying the control factors and driving factors of growth or decline in a city or group of cities, as well as growth tendencies in land use and the corresponding economic, social, and environmental effects. Good 'genes' from a city can be cloned to other cities in order to produce better urban forms, thus urban DNA contributes to the identification of critical factors on which urban characteristics largely relay, providing new insight and understanding.

Also, in this research, not only did spatial variables prove to be important for modeling the urban growth patterns as previous researchers stated regarding
DNA of our city, but also a-spatial dependent variables were taken into consideration. Urbanization is a multiscale process, and spatial variables alone cannot properly account for urban DNA or the unique characters of our city.

\section{References}

Ajzen, I. (1985). From intentions to actions: A theory of planned behavior. In J. Kuhl \& J. Beckman (Eds.), Action-control: From cognition to behavior, Germany: Springer.

Batty, M, Longley, P. (1994). Fractal cities. A Geometry of Form and Function, London: Academic Press.

Batty, M, Xie, Y. (1996). Preliminary evidence for a theory of the fractal city. Environment and Planning A: Economy and Space, 28(10):1745-1762.

Barthe'lemy, M, Flammini, A, (2008). Modeling Urban Street Patterns, Physical review letters, 100(13):1-4.

Caglioni, M, Pelizzoni, M, Rabino, G.A. (2006). Urban sprawl: a case study for project gigalopolis using SLEUTH model. Presented at 7th International Conference on Cellular Automata, for Research and Industry. France.

Clarke, K.C. (2008). Mapping and Modeling Land Use Change: an Application of the SLEUTH Model, in Landscape Analysis and Visualization: Spatial Models for Natural 
Resource Management and Planning, (Eds. Pettit, C., Cartwright, W., Bishop, I., Lowell, K., Pullar, D. and Duncan, D.), Berlin: Springer.

Franklin, G, Powell, J. D, Emami-Naeini, A. (2002). Feedback Control of Dynamic Systems (4 ed.). New Jersey: Prentice Hall.

Langton. C. (1986). Studying artificial life with cellular automata, Physical D: Nonlinear Phenomena., 22 (1-3):120-149.

Marshall, S. (2007). Urbanism in Evolution: New Urbanism and Beyond, retrieved from: https://www.cnu.org/sites/default/files/s marshall_cnu16.pdfurl.

Nicholas, G, Clarke, K. (2006). Exploring the DNA of our Regions: classification of outputs from the SLEUTH model. Presented at 7th International Conference on Cellular Automata, for Research and Industry. France.

Openshaw, S, Openshaw, C. (1997). Artificial intelligence in geography. New York: John Wiley and Sons.

Silva, EA. (2001). The DNA of our regions. Artificial intelligence in regional planning. presented in World Planning Schools Congress, Shanghai.

Silva, EA, KC, Clarke. (2002). Calibration of the SLEUTH urban growth model for Lisbon and Porto. Computer, Environment and urban systems, 26(6):525-552.

Silva, E.A. (2004). The DNA of our regions: artificial intelligence in regional planning, Futures, 36 (10):1077-1094.

Silva, EA, Clarke, K. (2005). Complexity, emergence and cellular urban models: lessons learned from Appling SLEUTH to two Portuguese cities, European Planning Studies, 13(1):93-115.

Silva, E A. (2006). Waves of Complexity: bifurcations or phase transitions, paper presented at IIIRD Meeting of AESOP thematic group on complexity. London.

Webster, C. (1996). Urban morphological fingerprints, Environment and Planning B: Planning and Design, 23:279-297.

Wilson, A. (2008). Urban and regional dynamics - 3: 'DNA' and 'genes' as a basis for constructing a typology of areas. CASA Working Paper 130. Centre for Advanced Spatial Analysis (UCL), London.

How to cite this article: Mohammad Rahim Rahnama, Alireza Bidkhori, Amirali Kharazmi, Analysis of Urban Planning Changes by Biological Metaphor (Case of DG-ABC Model). International Journal of Advanced Studies in Humanities and Social Science, 2019, 8(4), 384-391. http://www.ijashss.com/article_84388.html 